Please cite the published manuscript: Saad TC, Rodger D, Blackshaw BPResponding to objections to gatekeeping for hormone replacement therapy Journal of Medical Ethics Published Online First: 31 October 2019. http://dx.doi.org/10.1136/medethics-2019-105813

\title{
Responding to objections to gatekeeping for hormone replacement therapy
}

\section{Abstract}

In this brief reply to Ashley's rebuttal concerning the gatekeeping of hormone replacement therapy (HRT) for transgender people, we address some of the criticisms made of our original response to Ashley. We first re-examine Ashley's attempted analogy between abortion and HRT for gender dysphoria and find it wanting. Our argument that it is reasonable to regard cosmetic surgery and HRT for gender dysphoria as comparable (including in terms of prior psychological assessment) is restated to show that Ashley's previous objection to it is insubstantial. Our objection to Ashley's simultaneous rejection of the traditional informed consent model while maintaining its language, and thus relying on its positive connotations rather than argument, is then clarified. Finally, we scrutinise the data on which rests Ashley's claim that informed consent without assessment always yields positive outcomes, finding this conclusion to be overstated.

\section{Introduction}

Ashley has responded to our response to 'Gatekeeping HRT for transgender patients is dehumanising. ${ }^{1-3}$ Ashley criticises some of our objections to their view that patients seeking HRT for gender dysphoria should not have to undergo a prior psychological assessment. 1 Here, we clarify our objections, most importantly that concerning the parity between cosmetic surgery and the sort of intervention Ashley has in mind, showing that Ashley's criticism of our comparison is insubstantial. We start, however, by examining Ashley's comments regarding the analogy between abortion and HRT.After revisiting 
the subject of cosmetic surgery, we consider Ashley's use of the language of informed consent. We close by scrutinising the data on which Ashley relies to defend the claim that informed consent without assessment always yields positive outcomes, finding this conclusion to be overstated. Finally, even if Ashley's arguments were vindicated, the claim that the gatekeeping of HRT is motivated by hostility towards transgender people remains unsupported.

\section{Abortion and assessment}

Ashley objects to our calling their analogy between HRT for gender dysphoria and abortion strained, arguing that we have not explained why it is such. ${ }^{3} \mathrm{We}$ maintain that the analogy is strained and consequently undermines Ashley's objective. In arguing that neither pregnancy nor being transgender is an illness, Ashley likens abortion to interventions such as HRT-interventions which Ashley believes should not require prior psychological assessment. Yet, there are typically numerous restrictions surrounding abortion, and, importantly for Ashley's argument, some sort of psychological assessment is often a condition for the abortion to be authorised--for example, the referring doctor must believe that continuing the pregnancy will have a negative effect on the mother's mental health. Hence, Ashley's analogy is strained; abortion is different to their proposed model of transition-related interventions. Indeed, so different is abortion that invoking it seemingly reinforces the importance of psychological assessment, thus undermining Ashley's thesis. If, then, Ashley believes that induced abortion should no longer be subject to psychological assessment, then an argument should be made to this effect.

\section{Cosmetic surgery and stigma}

While acknowledging some similarities between cosmetic surgery and HRT for gender dysphoria, Ashley is unconvinced these have any bearing on transition-related interventions. Ashley draws attention to several possible differences between the two which 'may all amount to morally relevant differences'. ${ }^{3}$ Less hesitantly, Ashley adds that a significant moral difference is that people who undergo psychological assessments for cosmetic surgery 'are not stigmatised and pathologised in the way trans people are'. The argument is that people with gender dysphoria should not undergo a 
psychological assessment prior to transition-related interventions because they are stigmatised and pathologised.

This is a curious claim. Is it not likely that some people seeking cosmetic surgery feel and/or are stigmatised for their appearance? More importantly, why is this decisive? If, hypothetically, people with a cleft chin were stigmatised, and having such a chin came to be regarded as a medical problem to solve, why would this alone change the need for prior psychological assessment-let alone be dehumanising? Just because, for example, teenage pregnancy is stigmatised does not entail that women bearing such pregnancies are, therefore, dehumanised by undergoing a psychological assessment prior to an abortion.

Notwithstanding, we wonder if Ashley would support psychological assessment prior to HRT if society became wholly accepting of gender dysphoria, thus removing this factor from the equation. We speculate that Ashley would not, and so question the relevance of the appeal to stigma and medicalisation, which is the only concrete objection Ashley makes to our suggestion of parity between assessment for cosmetic surgery and transition-related interventions. Our previous point, therefore, that because HRT and cosmetic surgery are analogous, and cosmetic surgery is often preceded by psychological assessment, it is prima facie reasonable to treat HRT in the same manner. [Footnote $\left.{ }^{1}\right]$

\section{The 'informed consent' model}

We believe Ashley has mischaracterised our criticism of their arguments concerning the informed consent model, which may be due in part to a lack of clarity in our first response. Ashley's rebuttal helpfully underlines that they are deliberately moving away from the medical model. It thereby becomes clear that when Ashley is talking about 'informed consent', they do not mean it in the conventional sense, which presupposes a prior assessment (as per the medical model). Ashley is critical of our apparent failure to understand this: 'it is peculiar for Saad, Blackshaw, and Rodger to criticise me for adopting a model that is not compatible with the standard medical model'. ${ }^{3}$

\footnotetext{
${ }^{1}$ It is worth pointing out that there are other examples of where prior psychological assessment is recommended and considered routine prior to receiving treatment. One such example is bariatric surgery, which involves individuals who experience stigmatisation.7 The purpose of the psychological evaluation is to assess the patient's ability to manage the preoperative and postoperative demands of the surgery on account of the high prevalence of psychiatric morbidity in this population. 89
} 
This interpretation, however, misses our central criticism which, we admit, we did not adequately clarify. Our concern is that, while arguing for a paradigm shift in the management of gender dysphoria, Ashley persists in using the language of the rejected medical model, and thus benefits from the positive connotations that 'informed consent' has in its commonly understood context. Hence, it is not necessary to marshal a positive argument for Ashley's alternative model; the cachet of informed consent in the traditional model replaces an argument. Therefore, while we are acutely aware of Ashley's rejection of the medical model, we object that Ashley (and others) persist in using the language of the medical model, which they reject without making this strategy explicit.

\section{Evaluating the evidence for the 'informed consent' model}

Ashley notes in the original article and response that they provided two sources to support their claims that the informed consent model without prior assessment has yielded good outcomes. ${ }^{3}$ Whether this conclusion is fully supported by the evidence Ashley cites is what we question. For instance, Ashley backs up the claim by citing a study by Madeline Deutsch. ${ }^{4}$ However, 4 of the 12 sites surveyed in this study required patients to have input from a mental health provider, and, furthermore, 5 sites surveyed required a certain number of consultations prior to beginning HRT - which could also be construed as a kind of gatekeeping. This hardly seems to support the claim that prior psychological assessment is unnecessary, unethical and rooted in 'unconscious hostility towards trans lives and experiences'. 'It seems uncharitable to ascribe ill motive to healthcare professionals who practice and apply the medical model-a model that is still recommended under the World Professional Association for Transgender Health Standards of Care. ${ }^{5}$ Moreover, 7 of the 12 sites surveyed had been practicing the 'informed consent model' for less than 5 years. At one of those sites, $6 \%(n=10)$ of the patients were known to have expressed regret about HRT. The survey, however, only recorded known cases of regret and this was dependent on the sites' awareness and documentation or reporting of regret. It is, therefore, possible that regret was underreported. Regardless, even the lowest estimates of regret disprove Ashley's original claim that '...studies have shown no adverse outcomes associated with the informed consent model'.' 
Ashley's second source is a poster describing a retrospective chart review of the outcomes of patients aged 13-17 who accessed HRT using the 'informed consent model' ${ }^{6}{ }^{6}$ The limitations of the study detailed in the poster are clear: the sample size was small $(n=88)$, there are no details of a pilot test being undertaken and there was no comparison group. Although no adverse outcomes were recorded, as we argued in our first reply, it is premature and imprudent to remove all requirements for referral and assessment based on such limited evidence.

\section{Conclusion}

We hope that this response clarifies some of the points of contention between ours and Ashley's respective viewpoints. We believe we have shown that abortion does not set a precedent for interventions for non-pathological states without prior psychological assessment, and that Ashley's objection to our view that there is parity between HRT and cosmetic surgery is insubstantial. In revising the use of Ashley's 'informed consent' terminology, we clarified our concern that the positive connotations that such language has within the traditional model are giving Ashley's argument borrowed validity. We also examined some of the data Ashley's claims rely on and found Ashley's conclusions to be overdrawn. Finally, even if the arguments all stood up to scrutiny, Ashley still would have no evidentiary grounds to attribute ill motive to those who practise gatekeeping. It is vital that the open discourse needed to address so new and important an issue is not hindered by this sort of unfounded accusation.

\section{References}

1. Ashley F. Gatekeeping hormone replacement therapy for transgender patients is dehumanising. J Med Ethics 2019;45(7):480-2.doi:10.1136/medethics-2018-10529

2. Saad TC, Blackshaw BP, Rodger D. Hormone replacement therapy: informed consent without assessment?J Med Ethics:medethics-2019-105611.doi:10.1136/medethics-2019105611 
3. Ashley F. Reply to 'Hormone replacement therapy: informed consent without assessment?'. J Med Ethics:

medethics-2019-105628.doi:10.1136/medethics-2019-10562 8

4. Deutsch MB. Use of the informed consent model in the provision of cross-sex hormone therapy: a survey of the practices of selected clinics. Int $\mathrm{J}$

Transgend2012;13(3):140-6.doi:10.1080/15532739.2011.675 233

5. Coleman E, Bockting W, Botzer M, et al. Standards of care for the health of transsexual, transgender, and gender-nonconforming people, version 7 . Int $J$ Transgend2012;13(4):165-232.doi:10.1080/15532739.2011.7 00873

6. Blasdel G, Belkind U, Harris A, et al. Description and outcomes of a hormone therapy informed consent model for minors. Poster presented at the: 25th WPATH Symposium, Buenos Aires, Argentina, 2018.

7. Puhl RM, Heuer CA. Obesity stigma: important considerations for public health. Am J Public Health 2010;100(6):1019-28.doi:10.2105/AJPH.2009.159491

8. Snyder AG. Psychological assessment of the patient undergoing bariatric surgery. Ochsner J2009;9(3):144-8.

9. Wadden TA, Sarwer DB. Behavioral assessment of candidates for bariatric surgery: a patient-oriented approach. Obesitydoi:10.1038/oby.2006.283 